\title{
A GENERALIZATION OF ISEKI'S FORMULA
}

\author{
Pablo Panzone, Luis Piovan and Mariano Ferrari \\ Universidad Nacional del Sur, Argentina
}

\begin{abstract}
We prove a generalization of Iseki's transformation formula, which is basically a transformation formula for infinite products with certain variable exponents. We note that an infinite number of transformation formulae may be derived from this generalization and, as a corollary, a particular case is given.
\end{abstract}

\section{INTRODUCTION}

Sho Iseki's proof of the Dedekind transformation formula is well-known and appears in many textbooks $([1,12,14])$.

It uses the following fundamental formula which we recall here.

Theorem 1.1 (S. Iseki). Set

$$
\begin{aligned}
& \lambda(x):=-\log \left(1-e^{-2 \pi x}\right)=\sum_{m=1}^{\infty} \frac{e^{-2 \pi m x}}{m}, \\
& \Lambda(\alpha, \beta, z):=\sum_{r=0}^{\infty}(\lambda((r+\alpha) z-i \beta)+\lambda((r+1-\alpha) z+i \beta)), \\
& g(\alpha, \beta, z):=-\pi z\left(\alpha^{2}-\alpha+\frac{1}{6}\right)+\frac{\pi}{z}\left(\beta^{2}-\beta+\frac{1}{6}\right)+2 \pi i\left(\alpha-\frac{1}{2}\right)\left(\beta-\frac{1}{2}\right) .
\end{aligned}
$$

Assume that $0<\operatorname{Re}(z)$. If $0 \leq \alpha \leq 1$ and $0<\beta<1$, or, $0 \leq \beta \leq 1$ and $0<\alpha<1$, then

$$
\Lambda(\alpha, \beta, z)-\Lambda(1-\beta, \alpha, 1 / z)=g(\alpha, \beta, z) .
$$

2010 Mathematics Subject Classification. 11F20.

Key words and phrases. Transformation formulae, Iseki's transformation formula. Supported in part by UNS and CONICET. 
Notice the following trivial properties

$$
\begin{aligned}
& \Lambda(\alpha, \beta, z)=\Lambda(1-\alpha, 1-\beta, z), \\
& \Lambda(\alpha, \beta, z)=\Lambda(\alpha, \beta+1, z) .
\end{aligned}
$$

Below we give a generalization of the above formula. For this we introduce the function $\Lambda_{0}(\alpha, \beta, z, \xi, \theta)$, a generalization of Iseki's function $\Lambda(\alpha, \beta, z)$.

Our main result, Theorem 2.1, namely formula (2.2), is a generalization of Iseki's transformation formula (1.1). Indeed (1.1) can be easily recovered from (2.2) as explained in Remark 2.5.

There exists many transformation formulae in the literature related (or not) to modular forms. We do not pretend to be exhaustive but we mention for example [11, chapter 2], [13, chapter 4], and the papers [5-7] (the first paper contains abundant references up to 1977). S. Ramanujan has stated many wonderful transformation formulae in his notebooks. These have been proved thanks to B. Berndt work: see [2, chapters 14 and 15], [3, chapters 28, 30], and [4] and references therein. For example a very interesting generalization of a certain transformation formula of S. Ramanujan has been given on [3, page 327] in joint work with R. J. Evans. Also we mention the deep papers [8-10] where many modular functions and/or Hauptmodul for certain subgroups are given in product form.

Two points of interest concerning formula (2.2) are worth noting.

Firstly, formula (2.6) (see Remark 2.3) below shows that part of formula (2.2) is, with certain parameters, the logarithm of infinite products with certain variable exponents. Thus (2.2) may be seen as a transformation formula for this infinite product.

Secondly, if one differentiates (2.2) with respect to the variable $\theta$ then one obtains a new transformation formula (involving polylogarithms and other functions). This is explained in Remarks 2.5 and 2.6. A particular example of this, involving the dilogarithm, is given in the corollary below.

We discovered formula (2.2) when trying to obtain some kind of transformation formulae for products with (basically) a geometrical exponent. Our result, which we believe is new, is a more modest one and perhaps (though we do not know for sure) it is related to modular forms in some sense.

\section{A GENERALIZATION OF ISEKI'S FORMUla}

Set

$$
\gamma=\gamma(\theta):=e^{-2 \pi \theta}
$$

Using the abbreviation $e(x):=e^{2 \pi i x}$, we define

$$
\begin{aligned}
& \Lambda_{0}(\alpha, \beta, z, \xi, \theta):= \\
& \quad \sum_{m=1}^{\infty} \frac{\gamma^{\alpha} e^{-2 \pi m \alpha z} e(\beta m)}{\left(1-\gamma e^{-2 \pi m z}\right)(m+\xi)}+\sum_{m=1}^{\infty} \frac{\gamma^{\alpha-1} e^{-2 \pi m(1-\alpha) z} e((1-\beta) m)}{\left(1-\gamma^{-1} e^{-2 \pi m z}\right)(m-\xi)},
\end{aligned}
$$




$$
\begin{aligned}
& g_{0}(\alpha, \beta, z, \theta):= \\
& \frac{z}{\theta} \frac{e(\alpha \theta i)}{(e(i \theta)-1)}+\frac{e(-\beta \theta / z)}{(e(-\theta / z)-1)}\left(2 \pi i\left(\alpha-\frac{1}{2}\right)+\frac{2 \pi \beta}{z}\right)-\frac{2 \pi}{z} \frac{e(-\theta(1+\beta) / z)}{(e(-\theta / z)-1)^{2}} .
\end{aligned}
$$

Notice that for fixed $\alpha, \beta, \gamma, z$ such that $0<\operatorname{Re}(z), \theta \in \mathbb{C}, 0<\alpha, \beta<1$, $m \pm \xi \neq 0,1-\gamma^{ \pm 1} e^{-2 \pi m z} \neq 0$, if $m=1,2,3, \ldots$, then the right side of (2.1) converges absolutely (indeed, uniformly in the variable $z$ with the other variables fixed if we add $0<\epsilon<\operatorname{Re}(z))$.

Our main result is the following:

Theorem 2.1. Let $\Lambda_{0}$ and $g_{0}$ be defined by (2.1). If $0<\operatorname{Re}(z), \theta \in \mathbb{C}$, $0<\alpha, \beta<1$ then

$$
\Lambda_{0}(\alpha, \beta, z, \theta / z, \theta)-\Lambda_{0}(1-\beta, \alpha, 1 / z, 0,-i \theta / z)=g_{0}(\alpha, \beta, z, \theta) .
$$

The function $\Lambda_{0}$ is a generalization of Iseki's function $\Lambda$. Indeed

$$
\Lambda_{0}(\alpha, \beta, z, 0,0)=\Lambda(\alpha, \beta, z) .
$$

This follows from the definitions of the $\Lambda$ 's and

$$
\begin{aligned}
& \sum_{r=0}^{\infty} \lambda((r+\alpha) z-i \beta)=\sum_{r=0}^{\infty} \sum_{m=1}^{\infty} \frac{e^{2 \pi i m \beta}}{m} e^{-2 \pi m(r+\alpha) z} \\
& \quad=\sum_{m=1}^{\infty} \frac{e^{2 \pi i m \beta}}{m} e^{-2 \pi m \alpha z} \sum_{r=0}^{\infty} e^{-2 \pi m r z}=\sum_{m=1}^{\infty} \frac{e^{2 \pi i m \beta}}{m} \frac{e^{-2 \pi m \alpha z}}{1-e^{-2 \pi m z}}
\end{aligned}
$$

Some remarks are in order:

REMARK 2.2. The following properties, similar to those in (1.2), are easily seen from (2.1):

$$
\begin{aligned}
& \Lambda_{0}(\alpha, \beta, z, \xi, \theta)=\Lambda_{0}(1-\alpha, 1-\beta, z,-\xi,-\theta), \\
& \Lambda_{0}(\alpha, \beta, z, \xi, \theta)=\Lambda_{0}(\alpha, \beta+1, z, \xi, \theta) .
\end{aligned}
$$

REMARK 2.3. Notice the following closed form formulas for $\Lambda_{0}(\alpha, \beta, z, \xi, \theta)$ when $\xi=0,1 / 2$ in connection to formula $(2.2)$ (recall $\left.\gamma=\gamma(\theta):=e^{-2 \pi \theta}\right)$.

Lemma 2.4. Let $\theta$ be real, $0<\alpha, \beta<1$ and assume that $z$ is real, positive and large enough. Set $x_{n}:=x_{n}(\alpha, \beta)=e^{-2 \pi((\alpha+n) z-i \beta)} ; x_{n}^{\prime}:=x_{n}^{\prime}(\alpha, \beta)=$ $x_{n}(1-\alpha, 1-\beta)$. Then

$$
\begin{aligned}
\Lambda_{0}(\alpha, \beta, z, 0, \theta)=\log & \left(\prod_{n=0}^{\infty}\left(1-e^{-2 \pi((n+\alpha) z-i \beta)}\right)^{-\gamma^{n+\alpha}}\right. \\
& \left.\left(1-e^{-2 \pi((n+1-\alpha) z-i(1-\beta))}\right)^{-\gamma^{-n+\alpha-1}}\right),
\end{aligned}
$$




$$
\begin{aligned}
\Lambda_{0}\left(\alpha, \beta, z, \frac{1}{2}, \theta\right)= & -\frac{2 \gamma^{\alpha}}{1-\gamma}+\log \prod_{n=0}^{\infty}\left(\frac{1+\sqrt{x_{n}}}{1-\sqrt{x_{n}}}\right)^{\frac{\gamma^{\alpha+n}}{\sqrt{x_{n}}}} \\
& +\log \prod_{n=0}^{\infty}\left(\frac{1+\sqrt{x_{n}^{\prime}}}{1-\sqrt{x_{n}^{\prime}}}\right)^{\gamma^{\alpha-1-n} \sqrt{x_{n}^{\prime}}} .
\end{aligned}
$$

REMARK 2.5. We notice that the first terms of the Taylor series of the function $g_{0}$ in the variable $\theta$ around zero are given by

$$
\begin{aligned}
g_{0}(\alpha, \beta, z, \theta)= & g(\alpha, \beta, z)+\frac{\pi^{2} \theta}{6 z^{2}}(-4 i(\beta-1) \beta(2 \beta-1) \\
& \left.+(2 \alpha-1)(1+6 \beta(\beta-1)) z+2(\alpha-1) \alpha(2 \alpha-1) z^{3}\right) \\
& +O\left(\theta^{2}\right),
\end{aligned}
$$

where $g(\alpha, \beta, z)$ is the function defined in Theorem 1.1. Thus, thanks to (2.8) and (2.3), one recovers Iseki's formula (1.1) if one makes $\theta \rightarrow 0$ in (2.2).

The coefficients of the above Taylor's series of $g_{0}$ in $\theta$ are rational functions of the variables $z, \alpha, \beta$ (Note: This is easily seen from the wellknown expansion $\frac{z}{e^{z}-1}+\frac{z}{2}=\sum_{m=0}^{\infty} B_{2 m} \frac{z^{2 m}}{(2 m) !}$, where $B_{2 m}$ are the Bernoulli numbers). This is important due to the following remark.

REMARK 2.6. Formula (2.2) has the following nice property. Transformation formulas may be obtained in the following way: differentiate (2.2) $n$-times with respect to $\theta$ and then let $\theta \longrightarrow 0$.

Thus Iseki's formula (1.1), by Remark 2.5, is the first case, that is $n=0$, and the following corollary is the case $n=1$.

The following closed form formulas (2.9), (2.10) used in the corollary are proved together with it in section 5 (recall that the definition of the dilogarithm function is $\left.L i_{2}(z)=\sum_{n=1}^{\infty} \frac{z^{n}}{n^{2}}\right)$.

$$
\begin{aligned}
& \frac{\partial \Lambda_{0}(\alpha, \beta, z, 0,0)}{\partial \xi} \\
& =-\sum_{r=0}^{\infty} L i_{2}\left(e^{-2 \pi(\alpha+r) z} e(\beta)\right)+\sum_{r=0}^{\infty} L i_{2}\left(e^{-2 \pi(1-\alpha+r) z} e(1-\beta)\right)
\end{aligned}
$$

and

$$
\begin{aligned}
& \frac{\partial \Lambda_{0}(\alpha, \beta, z, 0,0)}{\partial \theta} \\
& =-2 \pi \alpha \Lambda(\alpha, \beta, z)+2 \pi \sum_{r=0}^{\infty} \lambda((r+1-\alpha) z-i(1-\beta)) \\
& \quad+2 \pi \log \left(\prod_{r=1}^{\infty} \frac{\left(1-e(\beta) e^{-2 \pi(r+\alpha) z}\right)^{r}}{\left(1-e(1-\beta) e^{-2 \pi(r+1-\alpha) z}\right)^{r}}\right) .
\end{aligned}
$$


Corollary 2.7. If $0<\operatorname{Re}(z), 0<\alpha, \beta<1$ then the following formula holds

$$
\begin{aligned}
& \frac{1}{z} \frac{\partial \Lambda_{0}(\alpha, \beta, z, 0,0)}{\partial \xi}+\frac{\partial \Lambda_{0}(\alpha, \beta, z, 0,0)}{\partial \theta}+\left(\frac{i}{z}\right) \frac{\partial \Lambda_{0}(1-\beta, \alpha, 1 / z, 0,0)}{\partial \theta} \\
& =\frac{\pi^{2}}{6 z^{2}}(-4 i(\beta-1) \beta(2 \beta-1)+(2 \alpha-1)(1+6 \beta(\beta-1)) z \\
& \left.\quad+2(\alpha-1) \alpha(2 \alpha-1) z^{3}\right)
\end{aligned}
$$

\section{Proof of Theorem 2.1}

The proof follows Iseki's proof closely. In a certain sense Iseki's proof is based on the identity

$$
\frac{1}{n(z n+i m)}=\frac{1}{i m n}+\frac{z}{m(m-i z n)} .
$$

Now making the change $z \rightarrow z+\theta / n$ gives the following formula

$$
\frac{1}{n(z n+i m+\theta)}=\frac{1}{i m n}+\frac{z}{m(m-i z n-i \theta)}+\frac{\theta}{n m(m-i z n-i \theta)} .
$$

This will be used later and it will be the heart of our proof.

We start with the following well-known Fourier expansion (here and elsewhere $\sum_{n}$ means $\lim _{N \rightarrow \infty} \Sigma_{n=-N, n \neq 0}^{N}$ ) valid for $0<m, 0<z, 0<\alpha<1$ ([Ise]):

$$
\frac{e^{-2 \pi \alpha z m}}{1-e^{-2 \pi z m}}-\frac{1}{2 \pi z m}=\frac{1}{2 \pi} \sum_{n} \frac{e(\alpha n)}{z m+i n} .
$$

Making the change $z \rightarrow z-\frac{\log (\gamma)}{2 \pi m}$, (where $\gamma:=\gamma(\theta)=e^{-2 \pi \theta}$ ), multiplying by $e(\beta m) / m$ and adding from $m=1$ to infinity gives

$$
\begin{aligned}
& \sum_{m=1}^{\infty} \frac{\gamma^{\alpha} e^{-2 \pi \alpha z m}}{\left(1-\gamma e^{-2 \pi z m}\right)} \frac{e(\beta m)}{m} \\
& \quad=\frac{1}{2 \pi} \sum_{m=1}^{\infty} \frac{e(\beta m)}{(z m+\theta) m}+\frac{1}{2 \pi} \sum_{m=1}^{\infty} \sum_{n} \frac{e(\alpha n+\beta m)}{m(z m+i n+\theta)} \\
& \quad=\frac{1}{2 \pi} \sum_{m=1}^{\infty} \frac{e(\beta m)}{(z m+\theta) m}+\frac{1}{2 \pi} \sum_{m=1}^{\infty} \sum_{n} A_{m, n, \theta, \alpha, \beta, z},
\end{aligned}
$$

where

$$
A_{m, n, \theta, \alpha, \beta, z}:=\frac{e(\alpha n+\beta m)}{m(z m+i n+\theta)}
$$


If in formula (3.3) one makes $\alpha \rightarrow 1-\alpha, \beta \rightarrow 1-\beta, \theta \rightarrow-\theta$ then one gets

$$
\begin{aligned}
& \sum_{m=1}^{\infty} \frac{\gamma^{-(1-\alpha)} e^{-2 \pi(1-\alpha) z m}}{\left(1-\gamma^{-1} e^{-2 \pi z m}\right)} \frac{e((1-\beta) m)}{m} \\
& \quad=\frac{1}{2 \pi} \sum_{m=1}^{\infty} \frac{e((1-\beta) m)}{(z m-\theta) m}+\frac{1}{2 \pi} \sum_{m=-1}^{-\infty} \sum_{n} A_{m, n, \theta, \alpha, \beta, z} .
\end{aligned}
$$

Thus, adding (3.3) and (3.4) and using the definition of $\Lambda_{0}$, we find that

$$
\begin{aligned}
\Lambda_{0}(\alpha, \beta, z, 0, \theta)= & \frac{1}{2 \pi} \sum_{m=1}^{\infty} \frac{e(\beta m)}{(z m+\theta) m}+\frac{1}{2 \pi} \sum_{m=1}^{\infty} \frac{e((1-\beta) m)}{(z m-\theta) m} \\
& +\frac{1}{2 \pi} \sum_{m} \sum_{n} A_{m, n, \theta, \alpha, \beta, z} .
\end{aligned}
$$

Now, using the following well-known formulas valid for $0<\beta<1$,

$$
\begin{gathered}
\sum_{m} \frac{e(-m \beta)}{m+\xi}=2 \pi i \frac{e(\beta \xi)}{e(\xi)-1}-\frac{1}{\xi},(\xi \notin \mathbb{Z}), \\
\sum_{m} \frac{e(-m \beta)}{m}=\pi i(2 \beta-1),
\end{gathered}
$$

one calculates

$$
\begin{gathered}
\frac{1}{2 \pi} \sum_{m} \frac{e(\beta m)}{(z m+\theta) m}=\frac{1}{2 \pi} \sum_{m=1}^{\infty} \frac{e(\beta m)}{(z m+\theta) m}+\frac{1}{2 \pi} \sum_{m=1}^{\infty} \frac{e((1-\beta) m)}{(z m-\theta) m} \\
(3.8)=\frac{1}{2 \pi} \sum_{m=1}^{\infty} \frac{e(\beta m)}{\theta}\left(\frac{1}{m}-\frac{1}{m+\frac{\theta}{z}}\right)+\frac{1}{2 \pi} \sum_{m=1}^{\infty} \frac{e(-\beta m)}{(-\theta)}\left(\frac{1}{m}-\frac{1}{m-\frac{\theta}{z}}\right) \\
=-\frac{2 \pi i}{\theta}\left(\beta-\frac{1}{2}-\frac{e(-\beta \theta / z)}{e(-\theta / z)-1}-\frac{z}{2 \pi i \theta}\right) .
\end{gathered}
$$

Set

$$
h_{\beta, z, \theta}:=-\frac{2 \pi i}{\theta}\left(\beta-\frac{1}{2}-\frac{e(-\beta \theta / z)}{e(-\theta / z)-1}-\frac{z}{2 \pi i \theta}\right) .
$$

Therefore the above formula (3.5) can be written more compactly as

$$
\Lambda_{0}(\alpha, \beta, z, 0, \theta)=h_{\beta, z, \theta}+\frac{1}{2 \pi} \sum_{m} \sum_{n} A_{m, n, \theta, \alpha, \beta, z} .
$$

Next we look at the last double sum of (3.9). Note that

$$
\sum_{m} \sum_{n} A_{m, n, \theta, \alpha, \beta, z}=\sum_{n} \sum_{m} A_{m, n, \theta, \alpha, \beta, z} .
$$


This can be proved as in [Ise] and its proof is left to the reader. Using this and (3.1), we find that

$$
\begin{aligned}
\sum_{m} \sum_{n} A_{m, n, \theta, \alpha, \beta, z}=\sum_{m} \sum_{n} A_{n, m, \theta, \alpha, \beta, z} \\
=\frac{1}{i}\left(\sum_{n} \frac{e(\beta n)}{n}\right)\left(\sum_{m} \frac{e(\alpha m)}{m}\right)+\sum_{m} \sum_{n} \frac{z e(\alpha m+\beta n)}{m(m-i z n-i \theta)} \\
\quad+\sum_{m} \sum_{n} \frac{\theta e(\alpha m+\beta n)}{n m(m-i z n-i \theta)} \\
=\frac{(2 \pi i)^{2}}{i}\left(\alpha-\frac{1}{2}\right)\left(\beta-\frac{1}{2}\right)+\sum_{m} \sum_{n} A_{m, n,-i \theta / z, 1-\beta, \alpha, 1 / z} \\
\quad+\sum_{m} \sum_{n} B_{m, n, \theta, \alpha, \beta},
\end{aligned}
$$

where we write

$$
B_{m, n, \theta, \alpha, \beta}:=\frac{\theta e(\alpha m+\beta n)}{n m(m-i z n-i \theta)}
$$

But

$$
\begin{aligned}
\sum_{m} \sum_{n} B_{m, n, \theta, \alpha, \beta} & =\sum_{n} \sum_{m} B_{m, n, \theta, \alpha, \beta} \\
& =\sum_{n=1}^{\infty} \sum_{m} B_{m, n, \theta, \alpha, \beta}+\sum_{n=-1}^{-\infty} \sum_{m} B_{m, n, \theta, \alpha, \beta} \\
& =\sum_{n=1}^{\infty} \sum_{m}\left(B_{m, n, \theta, \alpha, \beta}+B_{m, n,-\theta, 1-\alpha, 1-\beta}\right),
\end{aligned}
$$

and

$$
\sum_{n=1}^{\infty} \sum_{m} B_{m, n, \theta, \alpha, \beta}=\sum_{n=1}^{\infty}\left(\sum_{m} \frac{e(\alpha m)}{m(m-i z n-i \theta)}\right) \frac{\theta e(\beta n)}{n} .
$$

Also, we notice the formula

$\sum_{m} \frac{e(\alpha m)}{m(m-i z n-i \theta)}=2 \pi \frac{e^{-2 \pi \alpha(z n+\theta)}}{(z n+\theta)\left(1-e^{-2 \pi(z n+\theta)}\right)}-\frac{1}{(z n+\theta)^{2}}+\frac{\pi(2 \alpha-1)}{(z n+\theta)}$,

which will be proved later. 
Using (3.12), (3.13) and (3.14) one gets

$$
\begin{aligned}
\sum_{m} \sum_{n} B_{m, n, \theta, \alpha, \beta}= & 2 \pi \sum_{n=1}^{\infty} \frac{\theta e^{-2 \pi \alpha(z n+\theta)} e(n \beta)}{n(z n+\theta)\left(1-e^{-2 \pi(z n+\theta)}\right)} \\
& -2 \pi \sum_{n=1}^{\infty} \frac{\theta e^{-2 \pi(1-\alpha)(z n-\theta)} e(n(1-\beta))}{n(z n-\theta)\left(1-e^{-2 \pi(z n-\theta)}\right)} \\
& -\theta \sum_{n} \frac{e(n \beta)}{n(z n+\theta)^{2}}+\theta(2 \alpha-1) \pi \sum_{n} \frac{e(n \beta)}{n(z n+\theta)} .
\end{aligned}
$$

For these last two sums one uses (3.8) and the derivative of (3.8) with respect to $\theta$. For the first two sums one uses the definition of $\Lambda_{0}$ with the identity $\frac{1}{n}-\frac{1}{n+\theta / z}=\frac{\theta}{n(z n+\theta)}$, giving

$$
\begin{aligned}
\sum_{m} \sum_{n} B_{m, n, \theta, \alpha, \beta}= & 2 \pi \Lambda_{0}(\alpha, \beta, z, 0, \theta)-2 \pi \Lambda_{0}(\alpha, \beta, z, \theta / z, \theta) \\
& +2 \pi \theta \frac{\partial}{\partial \theta} h_{\beta, z, \theta}+\theta(2 \alpha-1) 2 \pi^{2} h_{\beta, z, \theta} .
\end{aligned}
$$

The chain of equalities (3.9), (3.11), (3.16) gives

$$
\begin{aligned}
\Lambda_{0}(\alpha, \beta, z, 0, \theta)= & h_{\beta, z, \theta}+\frac{1}{2 \pi} \frac{(2 \pi i)^{2}}{i}\left(\alpha-\frac{1}{2}\right)\left(\beta-\frac{1}{2}\right) \\
& +\frac{1}{2 \pi} \sum_{m} \sum_{n} A_{m, n,-i \theta / z, 1-\beta, \alpha, 1 / z}+\Lambda_{0}(\alpha, \beta, z, 0, \theta) \\
& -\Lambda_{0}(\alpha, \beta, z, \theta / z, \theta)+\theta \frac{\partial}{\partial \theta} h_{\beta, z, \theta}+\theta(2 \alpha-1) \pi h_{\beta, z, \theta} .
\end{aligned}
$$

Finally, canceling $\Lambda_{0}(\alpha, \beta, z, 0, \theta)$ from both sides in the above formula and using (3.9) one has

$$
\begin{aligned}
0= & h_{\beta, z, \theta}+\frac{1}{2 \pi} \frac{(2 \pi i)^{2}}{i}\left(\alpha-\frac{1}{2}\right)\left(\beta-\frac{1}{2}\right)+\Lambda_{0}(1-\beta, \alpha, 1 / z, 0,-i \theta / z) \\
& -h_{\alpha, 1 / z,-i \theta / z}-\Lambda_{0}(\alpha, \beta, z, \theta / z, \theta)+\theta \frac{\partial}{\partial \theta} h_{\beta, z, \theta}+\theta(2 \alpha-1) \pi h_{\beta, z, \theta} .
\end{aligned}
$$

This is formula (2.2) after a slight simplification.

We prove (3.14). Substract the following two identities

$$
\begin{gathered}
2 \pi \frac{e^{-a 2 \pi \alpha}}{\left(1-e^{-2 \pi a}\right)}-\frac{1}{a}=\sum_{m} \frac{e^{i m 2 \pi \alpha}}{a+i m}, \\
\pi-2 \pi \alpha=\sum_{m} \frac{e^{i m 2 \pi \alpha}}{i m} .
\end{gathered}
$$

This gives (3.14), if ones puts $a=z n+\theta$, and ends our proof. 


\section{Proof of Lemma 2.4}

Formulas (2.6) and (2.7) follow easily from the definition of $\Lambda_{0}$. Indeed,

$$
\begin{aligned}
\sum_{m=1}^{\infty} \frac{\gamma^{\alpha} e^{-2 \pi m \alpha z} e(\beta m)}{\left(1-\gamma e^{-2 \pi m z}\right) m} & =\sum_{m=1}^{\infty} \frac{\gamma^{\alpha} e^{-2 \pi m \alpha z} e(\beta m)}{m} \sum_{r=0}^{\infty} \gamma^{r} e^{-2 \pi m r z} \\
& =\sum_{r=0}^{\infty} \gamma^{\alpha+r} \sum_{m=1}^{\infty} \frac{e^{-2 \pi((\alpha+r) z-i \beta) m}}{m} \\
& =-\sum_{r=0}^{\infty} \gamma^{\alpha+r} \log \left(1-e^{-2 \pi((\alpha+r) z-i \beta)}\right) .
\end{aligned}
$$

Formula (2.6) follows easily from this.

Formula (2.7) follows similarly using the identities:

$$
\begin{gathered}
\sum_{n=1}^{\infty} \frac{x^{n}}{n+\frac{1}{2}}=-2+\frac{1}{\sqrt{x}} \log \frac{1+\sqrt{x}}{1-\sqrt{x}} \\
\sum_{n=1}^{\infty} \frac{x^{n}}{n-\frac{1}{2}}=\sqrt{x} \log \frac{1+\sqrt{x}}{1-\sqrt{x}} .
\end{gathered}
$$

5. Proofs of Corollary 2.7 and formulas (2.9) and (2.10)

The corollary, that is formula (2.11), is obtained differentiating (2.2) with respect to $\theta$, letting $\theta \rightarrow 0$ and using (2.8).

Formulas (2.9) and (2.10) are easy to prove. The proof of (2.9) is similar to $(2.4)$, and is left to the reader. To prove (2.10) one needs the identity

$$
\begin{aligned}
-\log \left(\prod_{r=1}^{\infty}\left(1-e(\beta) e^{-2 \pi(r+\alpha) z}\right)^{r}\right) & =\sum_{r=1}^{\infty} \sum_{m=1}^{\infty} \frac{r e^{2 \pi i m \beta}}{m} e^{-2 \pi m(r+\alpha) z} \\
& =\sum_{m=1}^{\infty} \frac{e^{2 \pi i m \beta}}{m} \frac{e^{-2 \pi i m(\alpha+1) z}}{\left(1-e^{-2 \pi m z}\right)^{2}} .
\end{aligned}
$$

ACKNOWLEDGEMENTS.

We are grateful to the referee for his careful reading and constructive remarks.

\section{REFERENCES}

[1] T. Apostol, Modular functions and Dirichlet series in number theory, Graduate Texts in Mathematics, No. 41. Springer-Verlag, New York-Heidelberg, 1976.

[2] B. Berndt, Ramanujan's Notebooks Part II, Springer-Verlag, New York, 1989.

[3] B. Berndt, Ramanujan's Notebooks Part IV, Springer-Verlag, New York, 1994.

[4] B. Berndt, Ramanujan's Notebooks Part V, Springer-Verlag, New York, 1998.

[5] B. Berndt, Modular transformations and generalization of several formulae of Ramanujan, Rocky Mountain J. Math. 1 (1977), 147-189. 
[6] B. Berndt, Generalized Dedekind eta-functions and generalized Dedekind sums, Trans. Amer. Math. Soc. 178 (1973), 495-508.

[7] B. Berndt, Generalized Eisenstein series and modified Dedekind sums, J. Reine Angew. Math. 272 (1975), 182-193.

[8] R. E. Borcherds, Monstrous moonshine and monstrous Lie superalgebras, Invent. Math. 109 (1992), 405-444.

[9] R. E. Borcherds, Automorphic forms on $O_{s+2,2}(R)$ and infinite products, Invent. Math. 120 (1995), 161-213.

[10] J. H. Conway and S. Norton, Monstrous moonshine, Bull. Lond. Math. Soc. 11 (1979), 308-339.

[11] H. Farkas and I. Kra, Theta constants, Riemann Surfaces and the Modular Group, Graduate Studies in Mathematics (AMS) 37, 2001.

[12] S. Iseki, The transformation formula for the Dedekind modular function and related functional equations, Duke Math. J. 24 (1957), 653-662.

[13] H. Iwaniec and E. Kowalski, Analytic Number Theory, Colloquium Publications (AMS) 53, 2004.

[14] H. Rademacher, Topics in Analytic Number Theory, Springer-Verlag, Die Grundlehren der mathematischen Wissenschaften 169, 1973.

\section{P. Panzone}

Departamento e Instituto de Matematica

Universidad Nacional del Sur

Av. Alem 1253, (8000) Bahia Blanca

Argentina

E-mail: ppanzone@uns.edu.ar

L. Piovan

Departamento e Instituto de Matematica

Universidad Nacional del Sur

Av. Alem 1253, (8000) Bahia Blanca

Argentina

E-mail: impiovan@criba.edu.ar

M. Ferrari

Centro Nacional Patagónico - CENPAT - CONICET

Bvd. Brown S/N, Puerto Madryn - CP 9120, Chubut

Argentina

E-mail: ferrari@cenpat.edu.ar

Received: 8.10.2009.

Revised: 17.12.2009. \& 21.1.2010. 